\title{
Synthesis and Analysis of a Simple Inductor-Less DC-AC Inverter
}

\author{
Kei Eguchi ${ }^{+}$, Kanji Abe and Wanglok Do \\ Department of Information Electronics, Fukuoka Institute of Technology, Japan
}

\begin{abstract}
To offer AC 100V from a DC 12V cigarette lighter socket, a simple inductor-less DC-AC inverter is proposed in this paper. In the proposed inverter, each capacitor is charged to the voltage of the preceding stage and then stepped-up by the voltage of the preceding stage increasing the voltage of the next stage. By connecting these capacitors in series, the proposed inverter offers AC $100 \mathrm{~V}$ by using smaller number of capacitors than conventional inductor-less inverters. Therefore, the proposed inverter can achieve small volume and light weight. The feasibility and effectiveness of the proposed inverter are demonstrated by SPICE simulations, theoretical analysis and experiments.
\end{abstract}

Keywords: DC-AC inverters, switched capacitor circuits, inductor-less circuits, Fibonacci sequence.

\section{Introduction}

A DC-AC inverter is a power device for changing DC to AC. For small power applications, the demand for a small and light inverter is increasing in recent years. The reason is common DC-AC inverters are heavy and bulky due to the existence of magnetic components. For this reason, an inductor-less DC-AC inverter is receiving much attention, because the inductor-less inverter can reduce not only circuit size but also effects of the electromagnetic interference (EMI). In 1998, Ishimatsu et al. suggested the voltage equational type inverter [1]. Following this, Oota proposed a bidirectional inverter [2] using a series-parallel type converter. Terada et al. suggested a programmable inverter using a ring type converter [3]. By controlling the timing of clock pulses, these inverters can offer not only a square wave but also a modified sinusoidal wave. However, these inverters require many circuit components, because the step-up gain of these inverters is proportional to the number of transfer capacitors. To reduce the number of circuit components, Chang suggested the multistage switched-capacitor-voltage-multiplier (SCVM) DC-AC inverter [4]. By connecting boost converters in series, the SCVM can achieve high gain. However, many circuit components are still necessary for the SCVM inverter, because the voltage ratio of all capacitors is the same.

In this paper, we propose a simple inductor-less inverter in order to achieve small size and light weight. The proposed inverter is operated in a three-phase manner. In the first- and second-phases, the voltage ratio of transfer capacitors becomes a Fibonacci number. In the third-phase, a high stepped-up voltage is generated by connecting these capacitors in series. By changing the polarity of the stepped-up voltage, the proposed inverter offers AC $100 \mathrm{~V}$ by using smaller number of capacitors than conventional inductor-less inverters. To clarify the characteristics of the proposed inverter, SPICE (simulation program with integrated circuit emphasis) simulations, theoretical analysis and experiments are performed.

\section{Circuit Configuration}

Fig. 1 illustrates the circuit configuration of the proposed DC-AC inverter. The proposed inverter is controlled according to the switching rule shown in Table 1 . In State- $T_{1}$ and $T_{2}$, the voltage of transfer capacitors becomes

\footnotetext{
${ }^{+}$Corresponding author. Tel.: +81-92-606-3137; fax: +81-92-606-0726.

E-mail address: eguti@fit.ac.jp.
} 


$$
V_{C(5-i)}=\sum_{j=3-i}^{4-i} V_{C j} \quad(i=\{0,1,2,3,4\}) \text {, where } V_{C 0}=V_{i n} \quad \text { and } \quad V_{C i}=0 \quad \text { if } \quad i<0 .
$$

In other words, unlike the conventional inverters such as series-parallel type inverter and voltage equational type inverter, the voltage ratio of capacitors becomes the ratio of a Fibonacci number. In State- $T_{3}$, all the capacitors are connected in series. Therefore, the $12 \mathrm{x}$ stepped-up voltage, $V_{o}$, is offered to the full bridge circuit. To generate an AC voltage $V_{\text {out }}$, the polarity of $V_{o}$ is changed alternatively by switching $S_{m}$ and $S_{p}$ at $50 / 60 \mathrm{~Hz}$. Of course, the proposed inverter can provide not only a square wave but also a modified sinusoidal wave by changing the number of series-connected capacitors in State- $T_{3}$. Table 2 shows the comparison of the number of circuit components between the proposed inverter and the conventional inverters. As you can see from Table 2, the number of circuit components for the proposed inverter is much less than that for the conventional inverters.

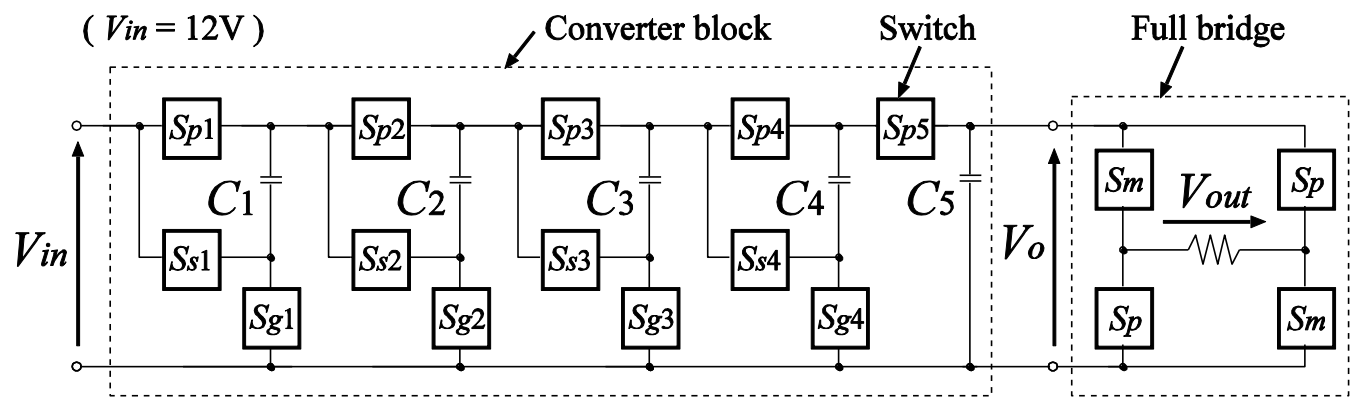

Fig. 1: Proposed DC-AC inverter.

Table 1: Switching rule in conversion ratio of 12

\begin{tabular}{|c|c|c|}
\hline State & On & Off \\
\hline$T_{1}$ & $S_{p 1}, S_{g 1}, S_{s 2}, S_{p 3}, S_{g 3}$ & Others \\
\hline$T_{2}$ & $S_{s 1}, S_{p 2}, S_{g 2}, S_{s 3}, S_{p 4}, S_{g 4}$ & Others \\
\hline$T_{3}$ & $S_{s 1}, S_{s 2}, S_{s 3}, S_{s 4}, S_{p 5}$ & Others \\
\hline
\end{tabular}

Table 2: Comparison of the number of circuit components

\begin{tabular}{|c|c|c|}
\hline Inverter type & Number of switches & Number of capacitors \\
\hline Proposed inverter & 17 & 5 \\
\hline Voltage equational type inverter [1] & 29 & 12 \\
\hline Series-parallel type inverter [2] & 38 & 12 \\
\hline Ring type inverter [3] & 48 & 12 \\
\hline Multistage SCVM [4] & 26 & 8 \\
\hline
\end{tabular}

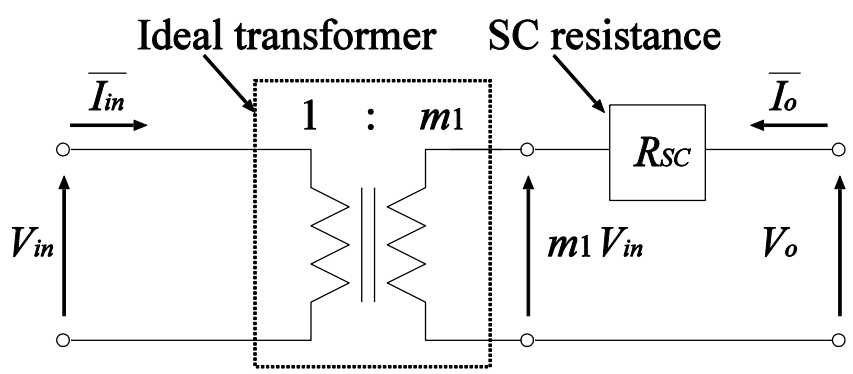

Fig. 2: Four-terminal equivalent model.

\section{Equivalent Model}

To clarify the characteristics of the proposed inverter, theoretical analysis is performed, where the equivalent circuit is assumed as a four-terminal equivalent model reported in [5]. Fig. 2 illustrates the fourterminal equivalent model, where $m_{1}$ denotes the ratio of an ideal transformer and $R_{S C}$ is called the SC (switched capacitor) resistance. In the theoretical analysis, we assume that 1) Transistor switch is modeled by an ideal switch with the on-resistance $R_{o n}$; and 2) Time constant is much larger than the period of clock pulses. Under these conditions, the instantaneous equivalent circuits of the converter block (see in Fig. 1) are 
expressed as Fig. 3.

In steady state, the differential value of the electric charge in $C_{k}(k=1,2,3,4,5)$ satisfies

$$
\sum_{i=1}^{3} \Delta q_{T i}^{k}=0, \text { where } T=\sum_{i=1}^{3} T_{i} \text { and } T_{1}=T_{2}=T_{3}=\frac{T}{3}
$$

In Eq. 2, $\Delta q_{T i}{ }^{k}$ denotes the electric charge of the $k$-th capacitor in State- $T_{i}$ and $T$ is the period of a clock pulse. From Fig. 3, the differential values of electric charges in $V_{i n}$ and $V_{o}, \Delta q_{T i, V i n}$ and $\Delta q_{T i, V o}$, are given as follows:

$$
\begin{aligned}
& \text { State- } T_{1}: \Delta q_{T_{1}, V_{\text {in }}}=\Delta q_{T_{1}}^{1}-\Delta q_{T_{1}}^{2}, \quad \Delta q_{T_{1}, V_{o}}=\Delta q_{T_{1}}^{5}, \quad \Delta q_{T_{1}}^{2}=-\Delta q_{T_{1}}^{3} \text { and } \Delta q_{T_{1}}^{4}=0, \\
& \text { State- } T_{2}: \Delta q_{T_{2}, V_{\text {in }}}=-\Delta q_{T_{2}}^{1}, \quad \Delta q_{T_{2}, V_{o}}=\Delta q_{T_{2}}^{5}, \quad \Delta q_{T_{2}}^{3}=\Delta q_{T_{2}}^{1}+\Delta q_{T_{2}}^{2} \text { and } \Delta q_{T_{2}}^{3}=-\Delta q_{T_{2}}^{4}, \\
& \text { State- } T_{3}: \Delta q_{T_{3}, V_{\text {in }}}=-\Delta q_{T_{3}}^{1}, \quad \Delta q_{T_{3}, V_{o}}=\Delta q_{T_{3}}^{4}+\Delta q_{T_{3}}^{5} \text { and } \Delta q_{T_{3}}^{1}=\Delta q_{T_{3}}^{2}=\Delta q_{T_{3}}^{3}=\Delta q_{T_{3}}^{4} .
\end{aligned}
$$

Using Eqs. 3 - 5, the average input current and the average output current can be expressed as

$$
\overline{I_{i}}=\frac{1}{T}\left(\sum_{i=1}^{3} \Delta q_{T_{i}, V_{\text {in }}}\right)=\frac{\Delta q_{V_{\text {in }}}}{T} \quad \text { and } \quad \overline{I_{o}}=\frac{1}{T}\left(\sum_{i=1}^{3} \Delta q_{T_{i}, V_{o}}\right)=\frac{\Delta q_{V_{o}}}{T},
$$

where $\Delta q_{V i n}$ and $\Delta q_{V o}$ are electric charges in the input and output, respectively. Substituting Eq. (2)-Eq. (5) into Eq. (6), we have the relation between the input current and output current as follows:

$$
\overline{I_{i n}}=-12 \overline{I_{o}} \text {, where } \Delta q_{V_{i}}=-12 \Delta q_{V_{o}} \text { and } \Delta q_{V_{o}}=\Delta q_{T_{3}}^{4} \text {. }
$$

From Eq. 7, the parameter $m_{1}$ in Fig. 2 is obtained as

$$
m_{1}=12 .
$$

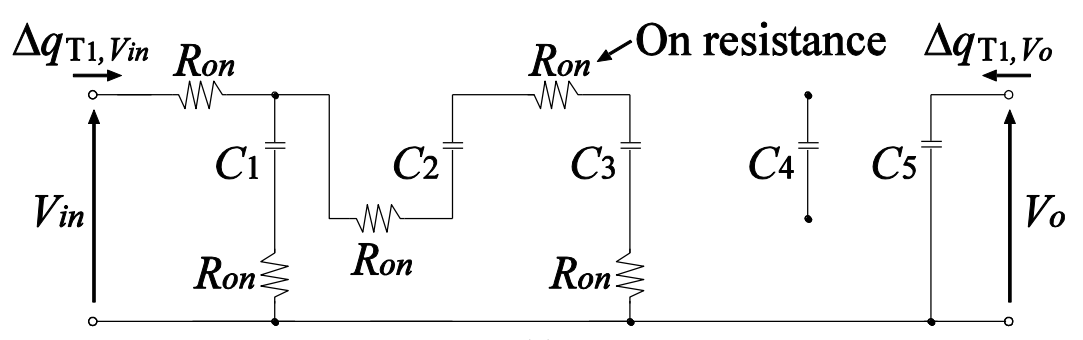

(a)

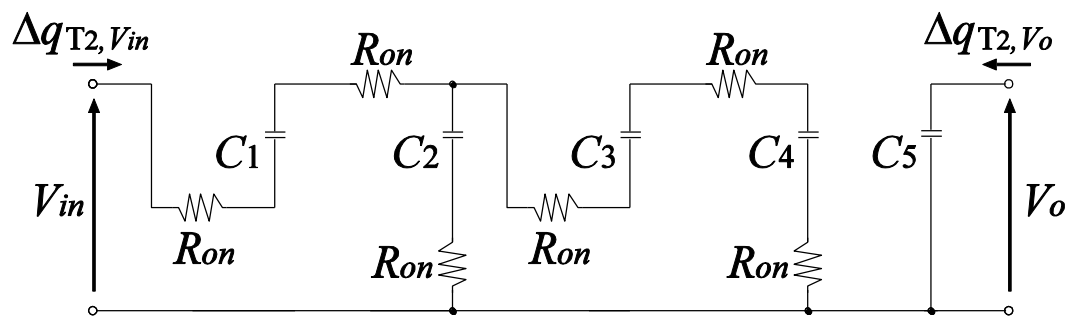

(b)

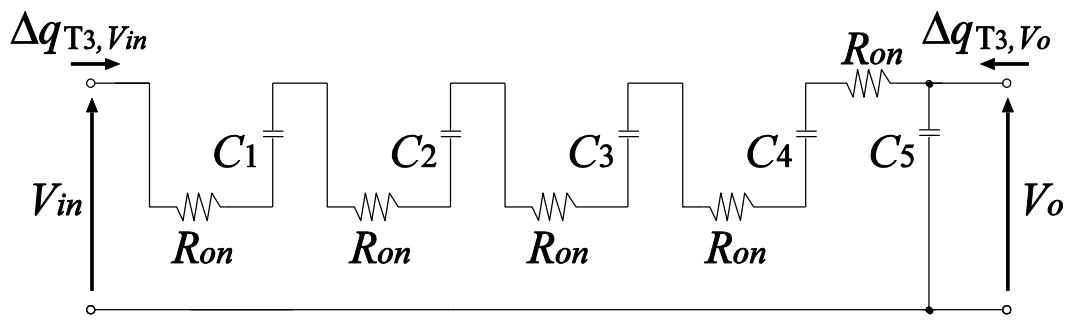

(c)

Fig. 3: Instantaneous equivalent circuits: (a) State- $T_{1}$, (b) State- $T_{2}$ and (c) State- $T_{3}$.

Next, in order to obtain the parameter $R_{S C}$, let us consider the consumed energy in one period. Using Eqs. 2 - 5, the total consumed energy can be expressed as 


$$
W_{T}=\sum_{i=1}^{3} W_{T_{i}}=\frac{405 R_{o n}}{T}\left(\Delta q_{V_{o}}\right)^{2}
$$

where $W_{T_{1}}=\frac{R_{o n}}{T_{1}}\left(\Delta q_{T_{1}}^{1}-\Delta q_{T_{1}}^{2}\right)^{2}+\frac{R_{o n}}{T_{1}}\left(\Delta q_{T_{1}}^{1}\right)^{2}+\frac{3 R_{o n}}{T_{s}}\left(\Delta q_{T_{1}}^{2}\right)^{2}$,

$$
W_{T_{2}}=\frac{2 R_{o n}}{T_{2}}\left(\Delta q_{T_{2}}^{1}\right)^{2}+\frac{R_{o n}}{T_{2}}\left(\Delta q_{T_{2}}^{2}\right)^{2}+\frac{3 R_{o n}}{T_{2}}\left(\Delta q_{T_{2}}^{4}\right)^{2} \text { and } W_{T_{3}}=\frac{5 R_{o n}}{T_{3}}\left(\Delta q_{T_{3}}^{4}\right)^{2} \text {. }
$$

On the other hand, the consumed energy of Fig. 2 can be expressed as

$$
W_{T}=\left(\frac{\Delta q_{V_{O}}}{T}\right)^{2} \cdot R_{S C} \cdot T .
$$

From Eq. (9) and Eq. (10), the parameter $R_{S C}$ is obtained as

$$
R_{S C}=405 R_{o n} \text {. }
$$

Finally, by combining Eq. (8) and Eq. (11), the four-terminal equivalent model of the proposed inverter can be expressed as Fig. 4. From Fig. 4, we have the equivalent circuit of the proposed inverter as follows:

$$
\left[\begin{array}{l}
\overline{V_{\text {in }}} \\
I_{\text {in }}
\end{array}\right]=\left[\begin{array}{cc}
\frac{1}{12} & 0 \\
0 & 12
\end{array}\right]\left[\begin{array}{cc}
1 & 405 R_{\text {on }}+2 R_{\text {on }} \\
0 & 1
\end{array}\right]\left[\begin{array}{l} 
\pm \overline{V_{\text {out }}} \\
-\overline{I_{\text {out }}}
\end{array}\right] .
$$

Therefore, we have the efficiency $\eta$ and output voltage $V_{\text {out }}$ as follows:

$$
\eta=\frac{R_{L}}{R_{L}+407 R_{\text {on }}} \quad \text { and } \quad V_{\text {out }}= \pm\left(\frac{R_{L}}{R_{L}+407 R_{\text {on }}}\right) \times 12 V_{\text {in }} .
$$

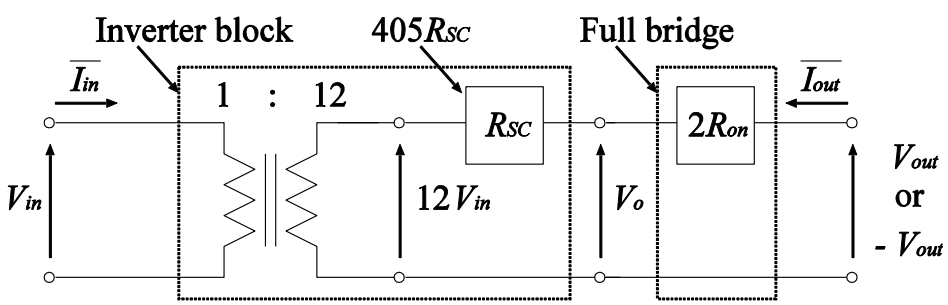

Fig. 4: Four-terminal equivalent model of the proposed inverter.

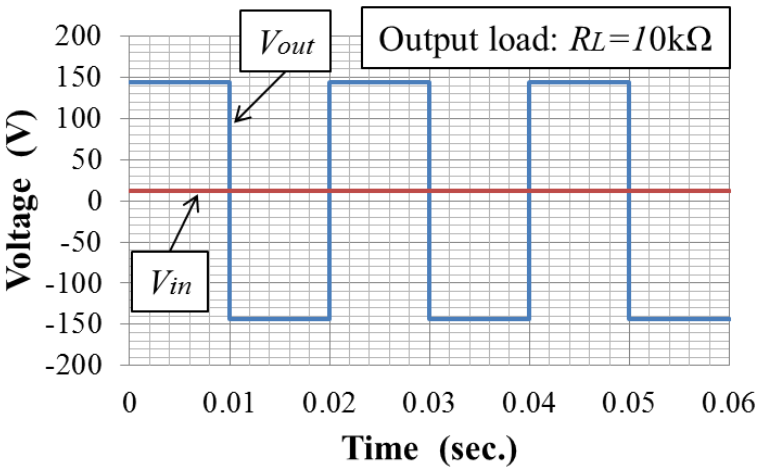

Fig. 5: Simulated output voltage.

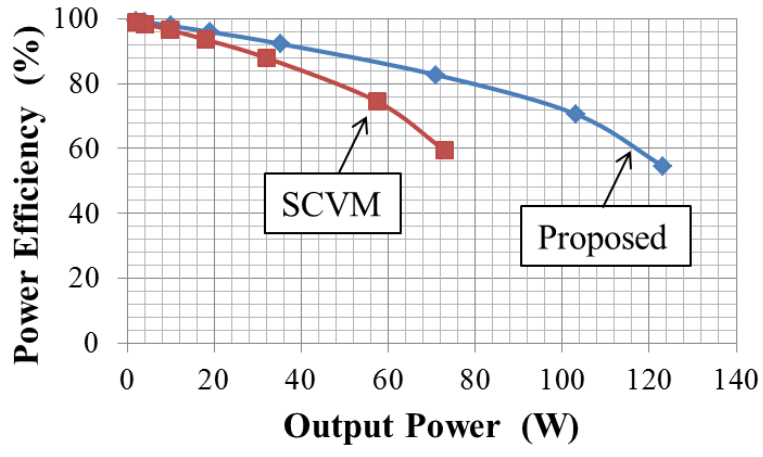

Fig. 6: Simulated power efficiency.

\section{Simulation \& Experiment}

Fig. 5 demonstrates the simulated output voltage of the proposed inverter. The SPICE simulation was performed under conditions that $V_{i n}=12 \mathrm{~V}, C_{k}=10 \mu \mathrm{F}(k=1, \ldots, 5), R_{o n}=0.1 \Omega$, and $T=1.5 \mu$ s. As you can see from Fig. 5, the proposed inverter offers AC $100 \mathrm{~V}$ by using smaller number of capacitors than conventional inductor-less inverters. Fig. 6 demonstrates the simulated power efficiency as a function of the output power. Of course, the output power can be increased by connecting the inverters in parallel. As you 
can see from Fig. 6, the power efficiency of the proposed inverter is higher than that of the conventional inverter [3]. Concretely, the proposed inverter can improve more than $9 \%$ power efficiency from the conventional inverter when the output power is $50 \mathrm{~W}$.

To confirm the validity of the proposed topology, experiments were performed. The experimental circuit was built with commercially available components AQV217 and TD62083APG on a breadboard, where $V_{\text {in }}$ $=12 \mathrm{~V}, C_{1}=\ldots=C_{5}=100 \mu \mathrm{F}, T=0.6 \mathrm{~ms}$ and $R_{L}=10 \mathrm{k} \Omega$. Fig. 7 (a) shows the measured output voltage of the experimental circuit. The experimental circuit was driven by the clock pulses shown in Fig. 7 (b). As you can see from Fig. 7, the proposed topology can offer about AC 100V output by converting the DC input $12 \mathrm{~V}$.

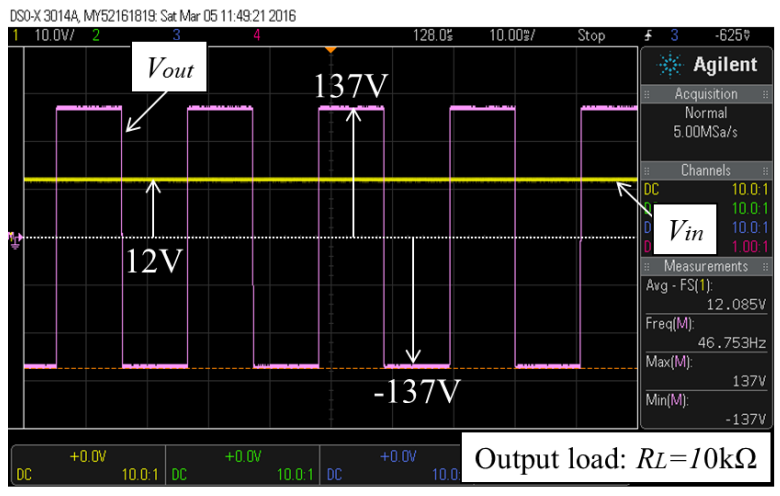

(a)

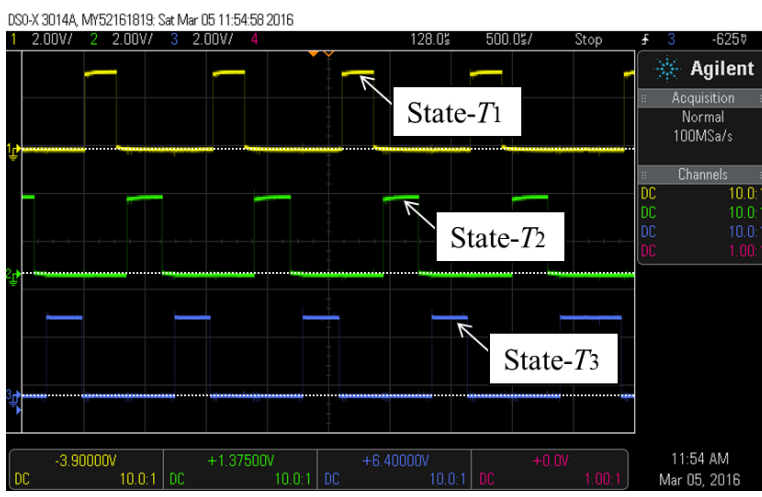

(b)

Fig. 7: Measured output voltage of the experimental circuit: (a) Output voltage and (b) Clock pulse.

\section{Conclusion}

A simple inductor-less DC-AC inverter has been proposed in this paper. By deriving a four-terminal model of the proposed inverter, the characteristics of the proposed inverter were clarified theoretically. The SPICE simulation and experiment demonstrated the following results: (1) The proposed inverter can offer AC 100V from DC 12V without magnetic components; (2) The proposed inverter can realize smaller size than conventional inductor-less inverters. Concretely, the proposed inverter can reduce more than $30 \%$ of circuit components from the multistage SCVM; and (3) The proposed inverter can achieve more than $80 \%$ power efficiency while the output power is less than $80 \mathrm{~W}$.

The IC implementation of the proposed inverter is left to a future study.

\section{References}

[1] K. Ishimatsu, I. Oota, and F. Ueno. A DC-AC converter using a voltage equational type switched-capacitor transformer. Proc. of the Thirteenth Annual Applied Power Electronics Conference and Exposition, APEC'98, Vol. 2. 1998, pp. 603-606.

[2] M. Oota, S. Terada, K. Eguchi, and I. Oota. Development of switched-capacitor bi-directional DC-AC converter for inductive and capacitive loads. Proc. of IEEE International Symposium on Industrial Electronics. 2009, pp. 1618-1623.

[3] S. Terada, I. Oota, K. Eguchi, and F. Ueno. A ring type switched-capacitor (SC) programmable converter with DC or AC input/ DC or AC output. Proc. of the $47^{\text {th }}$ IEEE International Midwest Symposium on Circuits and Systems. 2004, pp. I-29 - I-32.

[4] Y. H. Chang: Modelling and analysis of multistage switched-capacitor-voltage-multiplier boost DC-AC inverter. Proc. of IEEE 9th International Conference on Power Electronics and Drive Systems, PEDS2011. 2011, pp. 523526.

[5] K. Eguchi, P. Julsereewong, A. Julsereewong, K. Fujimoto, and H.Sasaki: A Dickson-type adder/subtractor DCDC converter realizing step-up/step-down conversion. International Journal of Innovative Computing Information and Control. 2013, 9 (3): 123-138. 